\title{
IDM as a "Minor" Literature: The Treatment of Cultural and Musical Norms by “Intelligent Dance Music"
}

\author{
RAMZY ALWAKEEL \\ UNIVERSITY OF LEEDS (UK)
}

\begin{abstract}
This piece opens with a consideration of the etymology and application of the term "IDM", before examining the treatment of normative standards by the work associated therewith. Three areas in particular will be focused upon: identity, tradition, and morphology. The discussion will be illuminated by three case studies, the first of which will consider Warp Records' relationship to narrative; the two remaining will explore the work of Autechre and Aphex Twin with some reference to the areas outlined above. The writing of Deleuze and Guattari will inform the ideas presented, with particular focus being made upon the notions of "minority", "deterritorialisation", and "continuous variation". Based upon the interaction of the wider IDM "text" with existing "constants", and its treatment of itself, the present work will conclude with the suggestion that IDM be read as a "minor" literature.
\end{abstract}

\section{Keywords}

IDM, Deleuze, minority, genre, morphology, subjectivity, Autechre, Aphex Twin, Warp

Can you really kiss the sky with your tongue in cheek?

Simon Reynolds (1999: 193)

Why have we kept our own names? Out of habit, purely out of habit. [...] Also because it's nice to talk like everybody else, to say the sun rises, when everybody knows it's only a matter of speaking. To reach, not the point where one no longer says I, but the point where it is no longer of any importance whether one says $I$.

Deleuze and Guattari (2004: 3-4) 


\section{Introduction}

Etymologically, an attempt to determine what "IDM" actually signifies is problematic. In August 1993, advertising a new group on the alt.rave Usenet mailing list, Alan Michael Parry suggested that

IDM (Intelligent Dance Music) is a forum for the discussion of what has been termed 'intelligent' music - that is music that moves the mind, not just the body. There is no specific defintion $[s i c]$ of intelligence in music, however, artists that I see as appropriate are FSOL, Orb, Orbital, Richard James (aka Aphex Twin), Blackdog [sic], B12, and various others from Warps $[s i c]$ artificial intelligence series.

Artificial Intelligence describes eight LPs released between 1992 and 1994 by Sheffield's Warp Records, billed in the sleevenotes to the first disc as "a series of 'listening albums" and "electronic music for the mind". The post does not clarify by whom this music "has been termed 'intelligent" but the reference to these releases suggests that the word's application may have been derived therefrom.

Awkwardly, though, the word "artificial" in the appropriated computer science term contests the intelligence in question. Further exploration of Warp's marketing accordingly reveals something of an ironic inconsistency to the series title. In the sleevenotes to the first album, the words "Artificial Intelligence" appear atop a consideration of the record's content that stipulates that "the atmosphere + emotion both come from the musicians", and that "their machines are merely the means to a human end". On the cover of the eighth release, however, the expression heads a far more transparent technical definition from the Encyclopaedia of Microcomputer Terminology that seems somewhat at odds with the first album's humanist dedication.

Rather than simply declaring the music's relationship to intelligence to be real as opposed to "artificial" (or the other way around), these texts challenge the validity of the artifice/authenticity binary itself. The role of the word "intelligence" in the alt.rave post becomes dubious: Warp's assertions that the music is "for the mind" and that "you weren't supposed to dance to it" (Reynolds 1999: 193) hardly justify an attempt to evaluate the level of intellect found therein. Indeed, when one studies the press material surrounding 1994's Artificial Intelligence II, it becomes clear that the word's employment by the Usenet group hinges upon something of a misreading. "Artificial Intelligence $[. .$.$] was never intended to be aimed at 'Intelligent' people (whatever they$ are)," it explains. "[It] was supposed to be a bit of a tongue-in-cheek dig at the people who said it was music made by computers that had no soul" (Young 2006: 62).

Artificial Intelligence thus appears to constitute something of a Socratic irony. Its title invites a reconsideration of the values attached to authenticity, intellect, and humanity, while the literature surrounding it challenges the assumption that these concepts should be either sacrosanct or central to its constitution.

In this article I will argue that, contrary to assertions by Simon Reynolds and others, the work associated with the term "IDM" bears no immediate relationship to hierarchical standards - neither those of delimited genres nor any of its own. Indeed, I will suggest not only that this work resists the term itself, but that, by so doing, it rejects the very notion of genre at all. I will conclude with a consideration of IDM in linguistic terms, with reference to Deleuze and Guattari's explorations of minority and majority. I will critique the idea that IDM affirms any sort of identity - even an ironic one - and suggest that, through its relationships with "the voice and law of tradition" (Colebrook 2002: 120), it can be read in some sense as a "minor" literature. 


\section{Canon}

Whilst not uncomplicated, it is clear that there exists some relationship between IDM and the forms and values of "tradition". I propose that this relationship is neither a straightforward embrace nor a straightforwardly ironic one that affirms the norms by throwing them into relief. Rather, in the next three subsections, I will consider IDM's treatment of canon as appropriative.

\section{Genre}

A minor literature does not write to express what it is (as though it had an identity to repeat or re-produce). [...] A literature would become majoritarian when its past examples are read as signs of some underlying essence (Colebrook 2002: 118).

Minority will return later. At this stage, I merely wish to open the debate about the applicability of Deleuze and Guattari's literary theory to our discussion by considering what we might understand in an IDM context by "past examples" being "read as signs of some underlying essence".

The original 1992 Artificial Intelligence sleeve depicts a robot, reclined in an armchair, facing a stereo system. On the floor are strewn three records: Pink Floyd's progressive classic The Dark Side of the Moon, Kraftwerk's groundbreaking Krautrock opus Autobahn, and Warp's first album release, a 1991 compilation entitled Pioneers of the Hypnotic Groove. It will be taken that the Floyd and Kraftwerk LPs signify a cultural "moment" in the context of the overall image; our focus now is the relationship of the third album cover to this moment, and to Artificial Intelligence itself.

The ideological effect of the appearance of the Pioneers sleeve on the $A I$ cover is not immediately clear. In a sense, it would seem to identify the former with the moment to which the other LPs allude, to construct Pioneers as influential, groundbreaking, canonical, and so forth. In isolation, this is awkwardly paradoxical: Dark Side and Autobahn predate both compilations by two decades. One possible response is to downplay the exclusive moment that supposedly connects Pink Floyd and Kraftwerk, but this removes their ability to function as a joint signifier, and invalidates any point made by their juxtaposition with Pioneers. If, alternatively, we ascribe to Pioneers the same level of groundbreaking influence as Autobahn and Dark Side, then any point made on the $A I$ cover becomes moot: its influences are of no interest or significance, because it is out-of-date: the IDM "moment" has already occurred.

We will see shortly that Warp's justification for the two older LPs' placement into the $A I$ text relates to their nature as records intended for listening purposes. However, Warp only introduced the "electronic listening music" epigram with $A I$ itself, whereas Pioneers of the Hypnotic Groove makes no attempt to align itself in this direction: most of its tracks are less rhythmically complex, more repetitive than those featured on $A I$, while "hypnotic" has easily as many connotations of trance music or of drug use at raves as it does of static listening.

A degree of humour could be contended here: we might subscribe to the idea that Warp is affirming its own debt to such "important" recordings by integrating itself ironically into their moment, as if to prove the absurdity of so doing. This, however, is inconsistent with the more academic self-awareness of the tone of the sleevenotes. Any irony remains Socratic: the viewer is confronted by a visual "problem" (this absurdity) that can only be "solved" by the critique of his or her own preconceptions (an examination of why the image is absurd). Artificial Intelligence's treatment of Pioneers calls 
into question the validity of distinction between pieces of music on the basis of any ascribed cultural significance, including that of perceived genre or "moment". We are invited instead to interrogate the ways in which the four albums - the three on its sleeve, plus its own fourth wall - are and are not related. The artwork challenges the notion that temporal proximity (between Pioneers and $A I$, the latter of which is surely the most representationally isolated of the four albums, since its invocation can only be reflexive) is any more valid a basis for equation than function ("listening music"). Rather than subscribing to a causal chain, the four records form interrelationships. The sleeve presents its viewer with an unpicking of the value system imposed by the idea of a canon of classics or of listening music (what is Pioneers doing in there?), or of an IDM canon itself (what are Floyd and Kraftwerk doing in there?). Not only is the music's involvement in any distinct genre resisted; interpreting the sleeve in light of the idea of a canon actually obscures its significance. "[H]umans organise or territorialise themselves through language. Language can then become inhuman or deterritorialised in art: no longer meaningful, controllable or recognisable" (Colebrook 2002: 59). The Artificial Intelligence artwork deterritorialises its own constituent signifiers. Genre and moment, inviolate in the language of the historical process, are seen no longer to be concrete, but to bear instead fluid relationships to a changing body of work - as with the addition of the Warp material, as equal, to Floyd and Kraftwerk.

This decentralisation is further demonstrated on Warp's second full-length release. LFO (whose eponymous first single appears on Pioneers) ask, in the sleevenotes to 1991's Frequencies,

What is house? Technotronic, KLF or something you live in. To me House is Phuture Pierre Fingers Adonis etc

The Pioneers of the Hypnotic Groove, Brian Eno, Tangerine Dream, Kraftwerk, Depeche Mode and the Yellow Magic Orchestra

The use of phrases like "What is house?" and "to me" indicate a strong emphasis upon democracy and discourse, formulating the delimitation of different "types" of music as arbitrary. Furthermore, the inclusion of Kraftwerk and Pioneers in the same list further blurs the distinction between "classic" albums and Warp's new output, especially given that LFO are effectively citing themselves in the latter.

Artificial Intelligence II strikes a further blow against closed definition. Within the album's gatefold is a quantity of text taken from an internet discussion thread about the $A I$ series' perceived similarity to and derivation from Detroit techno. No particular narrative or conclusion predominates, but two effects should be noted. Firstly, the inclusion of the thread itself elevates $A I$ s consumers to the level of $A I$ itself: the subtitle "More electronic listening music from Warp" appears on the same artwork as a public dissection of this very music. The argument about IDM's origins is thus seen to be democratic. Secondly, each successive post in the thread contains as much internal quotation as it does response; the emphasis is upon not exposition but dialogue. The argument about IDM's origins is thus seen to lack solution or authority to be, in Deleuze and Guattari's terms, rhizomatic (Deleuze and Guattari 2004: 3-28).

These texts, then, are perhaps more usefully considered outside of the conventional framework of genre The notion of genre is thus revealed to be incomplete: IDM cannot be hermetically sealed because it rejects authority and conclusion. It refuses to read "past examples [...] as signs of some underlying essence", because there is shown to be no unchanging ideal to which each successive release endeavours. 


\section{Progressive rock}

Reynolds makes several references to a connection between "intelligent techno" and the value systems surrounding the progressive rock of the 1970s. He identifies in various locations the "recast[ing of] progressive rock's Cartesian split between head and body as the struggle between atmospheric mind food (ambient) and thoughtless rhythmic compulsion (hardcore)" (Reynolds 1999: 184), "the unsightly flowering of the 'new progressive rock' that was always latent within the concept of electronic listening music" (Reynolds 1999: 200), and "a resurgence of rock notions like the 'concept album"' (Reynolds 1999: 182).

Elsewhere, he invokes the $A I$ packaging as a zone for the dance/don't dance binary. The initial 1992 compilation was presented in a gatefold sleeve - "a deliberate prog rock echo", he suggests (Reynolds 1999: 181) - whose design is outlined above. To illustrate his point, Reynolds (1999: 183) refers to Steve Beckett's statement that "we put [Autobabn and Dark Side] on the cover of Artificial Intelligence [...] to get it into people's heads that you weren't supposed to dance to it!" The semantics of the comment, however, are debatable. In the context of Beckett's earlier remark that Warp had largely grown out of his own experience of "realis[ing] [that certain tracks] weren't meant for twelve-inches, it was just that this was the only outlet for that kind of music" (Reynolds 1999: 183) it looks as though "you weren't supposed to dance to it" is less an instruction than the removal of one: music that does not require its consumers to dance if they choose not to. Not only does this set up a difficulty between IDM and its supposed genealogy as a type of dance music: it resists any unmediated incorporation into IDM of the progressive rock "text". This is but is not dance music: the locus of IDM lies neither in the dance lexicon nor in the rock lexicon, but in the tension between the two.

The argument around the music's use maps onto its definition. By resisting the prescription of its function, IDM is debarred from being theorised as a concrete genre.

When they turned their backs on the dance floor ('I see the term 'dance' as really restrictive for us", sniffed [Future Sound of London] member Brian Dougans) and dedicated themselves to concept albums, FSOL's pop instincts withered (Reynolds 1999: 200).

Reynolds' rejection of this lack of terminological complicity is highlighted by the connotations of pretension, pallor, even drug use in the word "sniffed", but it should be noted that the language of turning backs resides in the argument itself, and not Future Sound of London or their music: the identification of something as "restrictive" hardly necessitates the restriction of oneself to its other. Reynolds' attempt to incorporate IDM's resistance to definition back into the dualism he sets up is archaic: the texts challenge the value system itself, such that meaning now resides in the mediation of the rejection and embrace of "the term 'dance'". To assert that IDM (via Future Sound of London) has lost sight of its function as dance music is to attempt to close the linguistic sign after the horse has bolted.

Perhaps more pressing is the reference to a "split between mind and body", and the invocation of the auteur-subject. "For all its rhetoric of 'progression', intelligent techno involved a full-scale retreat from the most radically posthuman [...] aspects of rave music towards more traditional ideas about creativity, namely the auteur theory of the solitary genius who humanizes technology rather than subordinating himself to the drug-tech interface" (Reynolds 1999: 182). These concerns must be considered in light of the relationship, highlighted by the $A I$ rhetoric, between IDM and mecha- 
nism, and the implications upon subjectivity of the simultaneous mappings of mind and body onto "machine". It is to these that I will now turn.

\section{Humanism/mechanism; the mechanical subject-object}

A variety of IDM texts explore the meshing of human and mechanism, often by the location of "mechanical" forms within the human or of "subjective" forms within machinery. A thorough study of the interactions between IDM and posthumanism is clearly beyond the scope of the present work, but I nonetheless intend to provide a brief outline of some of the ways in which these texts approach the debate.

Below the definition of "artificial intelligence" taken from the Encyclopaedia of Microcomputer Terminology, the Artificial Intelligence II sleevenotes describe electronic music itself as "one bulletin board" upon which can be "posted" such questions as "do we still find mechanisation and commerce ugly?" and "is spirituality crushed beneath them?" Not only does this explicitly link a series that had seemingly claimed to be humanist in its conception (consider the quotations above from the $A I$ sleevenotes) with more progressive ideas about machinery; it democratises the debate by posing the problems as questions, not statements, and by the employment of the language of "bulletin boards" and "posting". This approach to the "problem" in turn hints at its mediation: by encouraging discussion rather than issuing an exposition, the sleevenotes invite dialectics into the conflict of ideology, and thus into the IDM text itself.

The debate is fleshed out, in part, by the explorations of "humanism" and "nature" that inform some of the work surrounding the notion of IDM. Distinctions are blurred as vocals are "spoken" by synthesised "robot" voices, as on the first track of LFO's Frequencies. Initially, this gesture suggests a distance between lyrics (taken from the album's sleevenotes quoted above) and author. Simultaneously, however, it sets up a unity between listener, author, and the machinic voice. All three are participating in the debate, but the author has lost authority, as the meeting is being chaired by the machine - which affirms its own subjectivity (using words like "me") and confronts the listener with its inauthenticity (it did not write the lyrics) in the same "breath". Conversely, the location of authorship within mechanism is also posited: LFO's very name - an abbreviation of Low Frequency Oscillator, a component in sound synthesis - identifies the music's human origins with its technological ones, while a similar effect is exacted by Harmonic $313^{1}$ - an alias of Mark Pritchard, who appears as part of Global Communication on Artificial Intelligence II. The latter band name likewise connects a typically "human" value (that of communication) to its technological means (the "global" phenomenon is unthinkable without the existence of telecommunications, high-speed transport, and so on).

Harmonic 313's album When Machines Exceed Human Intelligence might be considered, even with tongue in cheek, to re-establish a divide between "machines" and "human", but it should be noted that the latter is now being used as an adjective to describe one form of intelligence, and not an epithet that sets up machinic intellect as "artificial". The title can be seen as reference to an archaic projection founded upon such a divide: in being so titled, the record seems to immanentise this particular eschaton, but the album itself is in no tangible way "more" the work of machines than previous electronic releases. When Machines... again invokes the dialectic: it simultaneously affirms and denies the human/machine binary, refusing to locate authoritative value in either. 
Humanity is thus identified as both the object and the subject of technological development (compare LFO's robot/human blurring, and the resultant treatment of authorship, with $A \Gamma^{\prime}$ s rhetoric of human mastery over machines, or the complicity of When Machines Exceed Human Intelligence). This invalidates the pro-mind, prohuman agenda that Reynolds argues to be IDM's own, overturning both binaries through the introduction of a dialectical treatment of machine-subject and machineobject.

\section{Warp versus its Records: negative identity and dialectics}

Will Straw notes that disco labels throughout the 1970s

had little interest in developing long-term cumulative loyalty toward performers, and less still in building up back catalogues. [...] While the album had become, for the record industry, the dominant form through which a performer's identity found expression and assumed value, the lengthy periods normally required for its production and promotion were at odds with disco's rapid turnover and development (Straw 1999: 205).

Although not until 1989, it was to this commercial backdrop that Warp as a record label came together. Explaining its move to "foster [...] brand loyalty" (Reynolds 1999: 193 ) in an attempt to stay afloat, Reynolds cites Steve Beckett's observation that, around the period of Warp's formation, "dance labels had about a year of being on top. The only way to avoid that fate was to get more artist-oriented and album-oriented" (Reynolds 1999: 183).

Artificial Intelligence responded to this demand, showcasing six single-artist albums and reinforcing the brand with two bookending compilations upon which all of the six artists again appeared. The notion of a series at all inscribes the music - often presented as the work of oblique aliases or behind ambiguous album and track titles with more continuity of marketing than of content. Indeed, Warp's short films, generic purple record covers, and even television adverts might be seen to coalesce into a label identity stronger and more consistent than those of its individual records.

Nonetheless, Warp's roster never had any single overarching sonic agenda, encompassing both abstract electronic artists and guitar-oriented bands as early as 1990 (Young 2006: 14-16). The Artificial Intelligence debate around electronic music constituted just one of its "faces"; Rob Young cites Blech, a 1996 clubnight/ compilation complement, as part of "a distinguishable new phase" characterised by "a more human, fun side of electronica", a statement somewhat removed from the humanist politics surrounding $A I$. "By the late 90s", observes Young, "it was no longer possible to talk about a 'Warp sound' - it was more about a Warp state of mind” (Young 2006: 17).

However, even if Warp's identity is performed as a "state of mind" connecting elements of its output, the significance of these connections is in part negatively defined. " $[\mathrm{W}] \mathrm{e}$ might consider Brian Eno's claim that creativity now operates at the macro-level of whole genres, not at the micro-levels of individual artists. [...] [T] he entire scene $[. .$.$] is the creative force, producing collective movement in particular di-$ rections and leaving individual contributions to that movement to be seen as minor and transitory" (Straw 1999: 205-206). Warp's methods, then, are alien to much of the music in its catalogue, focusing not upon "scene" but upon albums and artists. At the same time, these artists have highly complex relationships to their own artistry, frequently engaging themselves discursively with identity politics. Warp's "state of mind" 
hinges upon its constituent artists and artworks, but the label refuses to conclusively brand anything under its umbrella, stylistically, canonically, or otherwise. A dialectical tension exists between the abstraction of the label's identity from its output and the very means to that output.

The case for any concrete relationships between texts in the Warp landscape is blown open by its tenth anniversary compilation series Warp 10, which comprises three collections: 10+1 Influences, 10+2 Classics 89-92 and 10+3 Remixes. Rob Young proposes that the albums demonstrate "a new self-awareness of the label as a participant in a specific music continuum and history" (Young 2006: 17), but this is only one side of the coin. As suggested above, Warp has already rejected specific history; "continuum" may, admittedly, be closer to the mark, but it is crucial that the three subtitles are seen not as fully constituted, distinct points in a narrative, but as potentials. The sticker on the front of $10+1$ Influences describes the record's content as "early house and techno", but this is far from the only area in which music released on Warp locates influence, as suggested by the diverse catalogue and the rejection elsewhere of the straightforward terminology of dance (consider the $A I$ debate). Meanwhile, $10+2$ Classics 89-92 compiles the label's first few single releases, but with a contradictory new subtext. The term "classics" seems to embrace the notion of canon, but this notion is precisely the one called into question by Pioneers of the Hypnotic Groove in the discussion above, even though both compilations repackage many of the same singles.

Indeed, it is significant that the latter two albums, despite bearing essentially the same tracklist, should perform two so different ideological functions - one disruptive and deterritorialising, the other operating within the language of hierarchy and precedent. This disjuncture reveals the tension from which Warp's "state of mind" is abstracted: once canon has been overturned, it can be used as a basis for the further dialectical exploration of artistic traditions and of the ways in which the texts might be read. We are invited to consider Warp's anarchic approach to linearity simultaneously with a text that claims identity precisely in this lack of identity. "[T] he more a language has or acquires the characteristics of a major language", write Deleuze and Guattari in this instance, the more IDM texts threaten to affirm their identity negatively - "the more it is affected by continuous variations that transpose it into a 'minor' language" (Deleuze and Guattari 2004: 113). The 1999 compilations do not, in fact, suggest any of the "underlying essence" denied by minor literature. Rather, by associating Warp with some of the values against which it was founded (recall the focus of the music identified by $10+1$ as "influence" upon singles over albums, and producers over artists (Straw 1999: 203-205)), Warp 10 places negative identity in "continuous variation".

It is, however, perhaps $10+3$ Remixes that reveals most explicitly the dialectical fragmentation of Warp's attitudes towards itself. Having branded - with whatever degree of irony - its first few pre- $A I$ singles "classics", Warp 10 concludes not with a retrospective covering its ascent into a significant portion of the alternative music market, but with an album of new remixes, all of whose source tracks come from Warp's back catalogue but many of whose remixers have never been associated with Warp at all.

A shift of focus takes place across these three albums. Both of the first two in fact feature 12-inch versions rather than album or 7 -inch mixes, even branding the tracks as such on the promotional stickers on their sleeves. In short, the fact that the tracks exist in different mixes (for example, the eponymous track by LFO appears in a version little over three minutes on the Frequencies LP and on its 7 -inch release, but the $10+2$ 
version is nearly six) is immaterial to the presence of the song texts themselves, in whatever form. ${ }^{2}$ However, the subtitle of the third collection, Remixes, brings the mixes rather than their originals into the foreground. The verb "to remix" is transitive: the original Warp catalogue becomes the object of these remixes, whose subjects are accordingly the non-Warp artists. By spotlighting the remixed artists in the first two instances, but the remixing artists in the third, Warp 10 makes the point that authorship is an interplay of context, not a singular concrete significance.

The effect of this is to downplay the connections that Warp 10 appears, upon first glance, to spin between points in Warp's continuum. Blurring the distinction between remixer and remixed (is the artwork located in the sound product such that the remixing subject is the artist, or in the creation of textual or notated "material" such that the object of the remix is the "real" artist?) dismisses the significance of Warp's back catalogue and history in precisely the same gesture by which it is celebrated - or, in fact, constructed.

In the first track on 10+3 Remixes, an LFO track and an Aphex Twin track are "remixed" into a single piece of music. Aphex Twin's 1994 album Selected Ambient Works Volume II features symbols in place of track names, and so a code appears on the $10+3$ tracklisting to identify the piece used: "SAW 2 CD1 Trk 7". SAW2's original lack of English titles is not read as a "[sign] of some underlying essence", but is treated as is most useful in the specific instance: nothing in the original text, even its extramusical elements, is sacrosanct. Elsewhere, a track "by" Autechre, "remixed" by Bogdan Raczynski, appears on disc two of 10+3, bearing the title "EP7/Envane". EP7 is in fact a collection of 12 tracks released by Autechre earlier the same year, totalling over an hour: formal distinctions are obscured by the presentation of ideas from across EP7s tracklist in a single piece of music. Envane, likewise, is a four-track collection from 1997. No Autechre-authored track entitled either "EP7" or "Envane" exists. The blurring of "EP" and "track" shows the forms of the original material to be protean; by appropriating its own constituent texts in this manner, Warp denies any component of its identity contingent upon these texts being immutable.

Minor languages are characterized [...] by a sobriety and variation that are like a minor treatment of the standard language, a becoming-minor of the major language. The problem is not the distinction between major and minor language; it is one of a becoming. It is a question not of reterritorializing oneself on a dialect or a patois but of deterritorializing the major language. (Deleuze and Guattari 2004: 116)

It is not sufficient that Warp perform the rejections of hegemony outlined above; for it then to read identity in the very process of so doing would demolish the texts' ability to deterritorialise anything. "Majority", write Deleuze and Guattari, "implies a constant, of expression or content, serving as a standard measure by which to evaluate it" (Deleuze and Guattari 2004: 116). To posit Warp's deterritorialising texts - such as the EP7 text that, we shall see below, itself calls into question the traditional distinctions between album and EP - as bearing an identity therein would be to territorialise them. In response, Warp 10's apparent celebration of identity deterritorialises that very identity. The process can only be dialectic: Warp rejects the formation of an identity consisting in the lack thereof but, at the same time, identity is re-appropriated and placed into continuous variation. 


\section{Fold4,Wrap5: Autechre versus morphological and linguistic convention ${ }^{3}$}

\section{Format}

Because of the industry within which Autechre operates, certain conventions of form are assumed. Catalogue numbers presuppose distinctions between albums and singles, as well as differences of physical format (7-inch, 12-inch, LP, cassette, CD, and so forth). Certain industry rules do not allow sound recordings to fall outside of these formal conventions: if a release is too long, it ceases to be eligible for the singles chart, for instance, while the British Phonographic Industry's barcoding guidelines allocate specific digits to different physical media (" 1 " for LP and " 2 " for CD, for instance). There is no option for a barcode not to feature exactly one of these digits (Official UK Charts Company 2009).

Autechre's approach to these physical conventions is inconsistent. 1999's EP7, by being so titled, allies its effects directly with those of the distinctions between formats: the "EP" affix suggests that the release is just this, while "7" identifies six of their previous releases in similar terms. However, counting its unlisted "hidden" track, the release features 12 pieces of music and totals nearly 67 minutes - greater in both respects than 1998 's $L P 5 .{ }^{4}$

This inconsistency can be read in at least two ways. Autechre's treatment of the album/EP division might be seen to recommend music's liberation from the morphology of record format altogether. The fact that EP7 has "EP" in its title does not make it shorter or less valuable than LP5. The use of the terminology could also be interpreted as its placement in "continuous variation". A distinction between album and $\mathrm{EP}$ on the basis of length is shown to be reductive; the various relativities and signifiers of which the distinction of "EP" is an aggregate do not function concretely. Demarcating an EP and an LP in spite of length when length is the industry's conventional discriminant detaches the terms from any absolute definition and subjugates them to the musicians' aesthetic decisions, deterritorialising them for use to signify whatever Autechre choose, in however abstract terms. This treatment of format "frees [it] from its actual origins" (Colebrook 2002: 58) (in this case, physical distinctions) so that it can be newly inscribed to whatever end its artists choose.

Format itself is not uniquely unstable: any artistic norm can be used in opposition to its original sense. Deleuze and Guattari identify "minorities" as "seeds [...] whose value is to trigger uncontrollable movements and deterritorializations of the mean or majority" (Deleuze and Guattari 2004: 117). As regards record format, EP7 is minor but, by so being, it reveals LPS - despite its apparent complicity with the standard "major" definitions - to be no less so, subverting the notion of concrete distinction that constructs $E P 7$ as deviant in the first place.

Elsewhere, the 1997 Cichlisuite EP blurs the "remix of"/"different song from" distinction by deriving its moniker from a track ("Cichli") that makes no clear connection to any of the EP's five parts - in terms either of title or of sonic content. The "Cichli" text is read subjectively: Cichlisuite's relationship to "Cichli" does not have to take the form of motivic or literary resemblance.

A more recent way in which Autechre's relationship to product challenges normative expectations can be identified in the release of the Quaristice album to digital download six weeks ahead of the physical LP and CD issues. "Our plan has never been to produce CDs - it's always been about making music. [...] The actual product is the FLAC file" (Park 2008a). The identification of "the product" at the point of the sonic 
material, not a physical object, draws attention to the transient quality of music; although this quotation refers specifically to the commercial, market download of Quaristice, the equation of product with data calls into question the abstraction of ownership from experience (of music). Although legal downloads have been accepted as a "format" for some time, it is unusual for their deployment to be primary - many catalogue numbers still identify a vinyl release as the "main" version of an album, denoting other formats by affixes. ${ }^{5}$

\section{Identification of the musical text}

As far as phonographic copyright is concerned, the musical text occurs at the point of those elements with the potential to be notated by conventional Western means: lyrics, melodies, chord progressions, and so forth. In short, these elements are taken to be "signs of some underlying essence" of the musical text as a whole, such that remixing or covering them does not detract from the cohesion of this text.

It has already been seen that Warp's attitude to remixing challenges these norms by reading different mixes as constitutive of new texts (as on 10+3 Remixes). The effect of this challenge is a play with the hierarchy of notation over sound. For now, I wish to consider one example of Autechre's treatment of this hierarchy.

The 1995 Garbage EP contains four tracks whose titles all end with a two-digit number. Upon closer inspection, the numbers denote the percentage of the EP's running time that each particular track makes up: "Vletrmx21", for instance, is 21 percent of Garbage's 40 minutes. This identification of the music's "visual" elements - track titles can surely be notated - with the broader sonic text serves to destabilise the hierarchy that discrete notation requires. Autechre directly links song title to the music's function not as a reduced set of "musical" signifiers but as a track, as the presence of recorded sound for a set number of minutes and seconds. The role that each track plays within the EP's running time is placed above the track's "notated" content sound in the purely spectrographic sense becomes central to the EP text, while any melodic, harmonic, or lyrical features are subordinated.

By working within such conventions as opus title, Garbage presents the listenerviewer with a challenge to the way in which musical texts are traditionally reduced. Replacing "Garbagemx36" with a remix that had a different running time but broadly the same "musical" content would transform the text in a way that remixing might not conventionally be considered to do. This elevates the mix, even the master, of a track to the same level as its more "visual" elements: the hierarchy is deposed.

\section{Literature}

Autechre might appear maddeningly cryptic, [with] titles [of tracks] like "Pen Expers" and "Bine" [...] Sean Booth [believes] their music is about evading all forms of "meaning", signification or representation (Stubbs 2003: 29).

The majority of Autechre's output has been identified by distorted technical terms, typographical errors, nonsense, or random selections of words. We might theorise an according relationship between opaque formations like "SonDEremawe", "90101-51-1", or "P.:NTIL" and the liberation of the letter from the word that drove, for instance, the work of the Lettrists in mid-20th Century France. The Lettriste Statement asserted that "the use of words in poetry is antiquated" (Cowley 2002: 199), a notion somewhat more forceful than Autechre's suggestion that "it was important for 
us not to pin down [Quaristice] to something too literal. [...] We're so into the music [that we] try not to colour it with any other ideas" (Park 2008b: 73), but the ideological work is related: both examples investigate the potential for operating outside of the sea of reference generated by the use of conventional words.

It is, of course, impossible to designate a linguistic space at zero potential, but the Autechre project is complicit with this: Sean Booth makes the point that "we quite like the ambiguity of the whole thing [...] People will always try and find meaning in something, so we let them" (Park 2008b: 73). What is of significance here is Autechre's awareness of the inefficacy of the attempt to circumscribe music using lexical reductions. The fact that Autechre use quasi-meaningless track titles is surely its own text, but it is a text that invites the listener-viewer to critique the role and value of title as reduction, much in the same way as Garbage's equation of title with spectrographic proportion calls into question what is expected from the literary component of a musical product. We recall that "humans [...] territorialise themselves through language", and that "[1]anguage can then become inhuman or deterritorialised in art: no longer meaningful, controllable or recognisable" (Colebrook 2002: 59). Although "language" and literature should not be equated - Colebrook's paraphrase does not deal specifically with the deterritorialisation of the letter itself - the rendering of written communication "no longer meaningful, controllable or recognisable" is certainly achieved by Autechre's quasi-"cut-up" approach to track titling.

Deterritorialisation of a sort thus occurs at the intersections between the Autechre project and the conventions of morphology that surround the production of musical texts - form, format, title. Without specifically denying any of the effects of these values, Autechre's treatment thereof invites a consideration of the extent to which such values are normally assumed to inform or be informed by the related music: the texts invoke debates around the relationship between form and content.

\section{Subjectivity}

Whether consisting abstractly in the text itself or in the fingerprints of an author, the location of the subject in music is necessarily a normative process. The abstraction of a subject at all requires a specific reading of certain textual elements in order to generate the text's relationship to distinct selves. Thus, Adorno's complaints about mass culture's treatment of subjectivity can be seen as analogous to, for example, Reynolds' identification of "the absence of heart and humanity" (Reynolds 1999: 185) in Autechre's work (although it is important to note that the quality of "emotional connection" of which Reynolds asserts Autechre's lack is far from what Adorno would consider an adequate relationship to the Geist).

Simon Frith (Frith 1988: 120) and John Shepherd (Shepherd 1999: 173) observe that the music industry responds particularly to music that bears "direct signs" of an authoring or performing subject (mediated, in these particular studies, through song lyrics, although this is not my focus here). This stage of the investigation will consider IDM's approach to such norms, with specific focus upon Aphex Twin's presentation of both product and identity. As Frith and Shepherd argue, popular music routinely transgresses the fourth wall in its dealings with subjectivity in such a way as to generate relationships between the author/performer and the listening subject. I propose that Aphex Twin's work breaks the fourth wall but affirms neither, revealing the space on both sides of the rupture to be awkwardly empty. I will suggest that the texts reveal a dialectical tension between Aphex Twin's location and absence in his 
own output, and, in so doing, discourage the placement of the subject atop any artistic hierarchy.

\section{Visual and acoustic space and subjectivity}

Marshall McLuhan and Erik Davis posit “visual space' [as] the dominant mode of Western consciousness following the Italian Renaissance" (Davis 2008: 53). This space is characterised with relation to a "visual self" (Davis 2008: 54) abstracted from such Euclidean thought processes (McLuhan 2007: 68) as "the power to create a distinct, single point of view that organizes thought and perception along linear lines" (Davis 2008: 54). This power derives from, for instance, perspective painting, print technologies, and the linearity of ordering language into a "phonetic" alphabet - a "mode of representation having neither visual nor semantic meaning" (McLuhan 2007: 71) that "promotes the illusion of removing oneself from the object" (McLuhan 2007: 69). Davis identifies a key feature of this visual mode of consciousness as "the axiom of assumption that 'different' objects, vectors or points are not and cannot be superimposed [such that] the world is perceived as a linear grid organized along strictly causal lines" (Davis 2008: 54).

McLuhan contrasts visual space with an "acoustic space" that "emphasizes simultaneity" and is "multidimensional, resonant, invisibly tactile, 'a total and simultaneous field of relations"' (Davis 2008: 54). Referring to studies by Edmund Carpenter, Bronislaw Malinowski and Dorothy Lee, he suggests that the perception of causality is a product of Western literacy and visual subjectivity (McLuhan 2007: 70), a hierarchy weighted unreasonably in favour of "the very physiology of the eye" that seems to debar any two things being in the same place at once, and instead "promotes the idea that everything is in sequence" (McLuhan 2007: 69). Acoustic space, however, "unlike visual space, where points either fuse or remain distinct", permits "blocks of sound [to] overlap and interpenetrate without necessarily collapsing into a harmonic unity or consonance, thereby maintaining the paradox of 'simultaneous difference" (Davis 2008: 54).

It is not necessarily the case that every acoustic phenomenon automatically subscribes to an acoustic mode of conceptualisation: McLuhan's analysis of the "phonetic" alphabet firmly roots it in a visually generated distance from the object, while the subject-relationships described by Frith and Shepherd separate the author and listener from the text and from each other. I now wish to demonstrate that IDM is insufficiently accounted for by the traditional value bestowed upon visual modes of thinking.

\section{"Acoustic" treatment of the subject in time}

I wish [Aphex Twin, Plastikman, Scanner and Daniel Pemberton] would not allow themselves any repetitions, and would go faster in developing their ideas or their findings, because I don't appreciate at all this permanent repetitive language. It is like someone who is stuttering all the time, and can't get words out of his mouth (Stockhausen, in Stockhausen et al. 2007: 382).

I disagree about repetition: I think, as John Cage said, repetition is a form of change (Scanner (Robin Rimbaud), in Stockhausen et al. 2007: 384).

Putting to one side the linguistic component of Stockhausen's point - "stuttering" is certainly an image to which Deleuze refers - I shall consider, briefly, some of the subjective values attached to repetition and change. 
"Change" implies narrative; narrative implies abstraction: for something to be identified as changing, it must possess a component that is perceived to be constant, or its different states would simply be different things. To say that something has "changed into" something else is to link the two together. Stockhausen's response to IDM is visual: it values linearity and development, both of which presuppose a musically mediated subject in the sense that Schopenhauer identifies:

In melody, in the high, singing, principal part, which dominates the whole and progresses freely in a single, uninterrupted, coherent and meaningful idea from start to finish, a complete entity in itself, I recognise [...] the conscious life and strife of man (Schopenhauer 1988: 221).

The isolation of this "idea from start to finish" accords with Stockhausen's observation that the work of IDM artists engenders a lack of "progression" - indeed, both writers make use of the term. The attachment to "the conscious life and strife of man" suggests that removing or transfiguring this "single, uninterrupted, coherent and meaningful idea" might also remove or transfigure the music's subjectivity. The equation of the two goes some way to illuminating Stockhausen's image of a person stuttering, unable to communicate: Stockhausen's subject is single and linear, so repetition or development is recorded against a single, linear axis.

This stuttering is only communicative failure if we are looking to music to provide us with a narrative. Alternatively, we can relate repetition to IDM's potential use as dance music: "I think [Stockhausen] should listen to a couple of tracks of mine [...] then he'd stop making abstract, random patterns you can't dance to" (Aphex Twin (Richard D. James), in Stockhausen et al. 2007: 383). This is not to say that Aphex Twin's work affirms the identity of "dance music" and therefore operates under its own normative system - many Aphex Twin tracks are not, in fact, particularly repetitive, and the debate about dance/listening as function has already been addressed. Rather, Richard D. James' point juxtaposes dance norms with a type of music that is identified later in the same interview as inappropriate to this function. Exclusively functional and exclusively subjective analyses are both rejected; theoretical "canon" carries no weight, so Stockhausen's music is necessarily open to constant re-assessment in light of new or evolving modes of use.

Adorno asserts that repetition of material is not repetition of its effect (Adorno 1988: 103). This relies for its truth upon the designation of a continuous, unidirectional time axis, allied with the experience of the visual subject. Repetition, in this language, cannot be total, because the time value will always be different: an event, in four-dimensional spacetime, can never be replicated. In Aphex Twin's language, however, repetition need not connote lack of fulfilment. Cycling units do not have to be seen as an undeveloping sequence documenting the subject's movement in time through the music, but might be devoid of a linear subject altogether, and therefore exist to some degree outside time; Stockhausen's assertion that "using music like a drug is stupid" (Stockhausen et al. 2007: 382) betrays a refusal to think outside of the values from which drugs might be a removal, of which conventional attitudes to time and self could easily be an element.

Repetition is only identified as such if the music is experienced in a single, linear dimension. Scanner's point that "repetition is a form of change", far from agreeing with Adorno, suggests that the experience of music from the perspective of a visual subject unreasonably precludes alternative modes of its perception and use. 


\section{"Acoustic" treatment of the subject in space}

By "space", I refer to voicing, to what in notational terms is described by "vertical": simultaneity. Essentially, all recorded sound is the object of McLuhan's "simultaneous difference" because of the equivalence of a single, complex waveform to the overlaying of a number of simpler ones, and the nature of sound vibrations as periodic and vector: it is possible to hear, as McLuhan points out, more than one sound distinctly and simultaneously - indeed, rarely if ever are we not doing so.

Nonetheless, like McLuhan's phonetic alphabet, there are traditions that eliminate this efficacy by identifying the musical text at the point of, for instance, notation itself. Linking notes to distinct instruments on a score prematurely resolves the paradox of simultaneous difference by spatially separating the different instrumental parts. Recordings of scored music, where they are identified as such, subscribe to the same system: regardless of the simultaneous difference in the waveform, what is represented is notational distance.

Schopenhauer's reference to a "principal part" is one result of this visual mode of hearing. His analysis renders time merely another spatial dimension: the parts are no more simultaneous than events that occur on the same axis of height, width or depth. For music to emphasise simultaneous difference, then, it must resist its reduction into visually distinct parts, especially parts arranged in a hierarchy (with a "principal").

IDM's rejection of reduced "material" has already been discussed. Clearly, I wish to avoid theorising any canon of technique around IDM for the reasons outlined above, but much of the music discussed in this article can be openly analysed in acoustic terms: nothing even contentiously associated with the term "IDM" has ever been published and marketed in sheet music form. It is dangerous, at this stage, to distinguish between material and technique, between content and form, but the emphasis of much of the music away from part-writing means that its reduction into distinct lines for distinct instruments - even distinct synthesiser patches - would often be virtually impossible. ${ }^{6}$ Given these conditions, the sonic presence of what could be identified as a melody or a bass line does not automatically demarcate them as such. When an artist who has at some stage worked with what seems to be melody creates a track consisting entirely of what seem to be percussion samples (such as Polygon Window's "Quoth") then the purpose of defining either in concrete terms becomes unclear. The frequency of such irregularities of "focus" in IDM necessitates the reconsideration of vertical hierarchy and disables visual subjectivity, reliant in Schopenhauer's terms as it is upon the distinction between "parts". The speaking subject is called strongly into question even apart from the treatment of actual vocal samples, which are few and far between, and whose looping and processing firmly distances them from any reading as signifiers of unmediated subjectivity.

\section{Aphex Twin and the fourth wall}

It is not, however, necessarily the case that the authoring subject is absent from the IDM text altogether, merely that "visual" analyses thereof are insufficient. In this subsection, I will look at the ways in which Aphex Twin's subjectivity - in the form of, or distinct from, that of Richard D. James - is treated by the employment of humour and irony in his work. I wish to focus primarily on the way that the Aphex Twin material is packaged and presented but, first, a brief look at one of the vocal samples to which I allude above will be illuminating. 
“To Cure a Weakling Child”, from 1996's Richard D. James Album, contains a sample of James himself, processed to sound like the voice of a child, speaking a list of body parts (James and Weidenbaum 2001). What is the effect of this use of the human voice? Certainly, the sample's semantic content does not mark James' subjectivity any more immediately than the "instrumental" material that surrounds it, and its processing makes it difficult to read as a signifier of the human artist. If anything, it might appear to locate humanity's role in the music as subordinate to the technology surrounding it; at the very least, it begins to "deterritorialise" the voice, to subject it to what the Situationists referred to as "détournement" (Poschardt 1998: 263-264).

It is, however, possible to locate subjectivity here, albeit dialectically. "To Cure a Weakling Child" features James' voice, but it might as well be an actual child, or a synthesis. James makes use of his own body not to master his equipment and techniques but to provide them with material - to become their object. At the same time, James himself undertakes the processing of this material, and chooses not to reference the voice of the author. His subjectivity is negatively defined: the vocal treatment shatters the illusion that vocals are a direct communiqué between vocalist and listener and, in so doing, opens up other areas for James to perform or distort his identity.

I now wish to look at some of the literature and artwork surrounding James' recorded output. As mediator between the music and the consumer, much of Aphex Twin's packaging has a complicated relationship to the music industry. The "Come to Daddy" single, for example, presents an intricate play between the multiple layers of representation upon which the reproducible work of art must simultaneously operate. The track was launched off the back of its use in an Orange TV advert, but the product's relationship to this fact is complex, endorsing and distancing the brand simultaneously ("the future is bright, the future is a registered trademark", notes the back of the 12-inch sleeve). A promotional edition of the single replaces all the artwork of the commercial formats with written descriptions: the line "a picture of school children with my face superimposed over their own" adorns the inner sleeve, for instance.

The artwork for Richard D. James Album invokes a similar tension between industry and subjectivity: the back cover replicates a tracklist hastily scribbled by hand, the proportions poorly thought out and a couple of crossings-out remaining. The front bears a weirdly processed photograph of James himself. An extreme close-up of the handwritten tracklist appears on the side B centre label, with only a couple of words legible. The album title itself, meanwhile, locates the musical content around James' own subjectivity, but the album is anything but straightforwardly personal "To Cure a Weakling Child" opens the second side, while other tracks have such opaque titles as "Goongumpas" and "Peek 8245452,01".

Both artwork and title make a case for Aphex Twin's dialectical subjectivity. The seemingly "personal" attitude that suffuses the use of his "real" name and the incorporation of his handwriting is presented simultaneously with the lack of subjectivity therein: the distance between author and listener is highlighted by the elevation of the mechanical means to their mediation, as with the pixellation of the blown up handwriting on the record label, or the processing of James' voice in the recording. At the same time, James himself has subjective control over these means: he himself has chosen to bring out the impersonal mediations. We are again confronted by the mechanical subject-object.

There is no "message" behind literary affect and becoming; any sense of a message or an underlying meaning is an effect of specific styles. It is the mask that produces the effect of a 
speaker behind the mask, but one can only speak because there are masks or personae (Colebrook 2002: 120-121, italics in original).

James breaks the fourth wall by drawing attention to these masks - the irrelevance of titling this particular album with his own name, the computer processes that render his handwriting and voice available to the listener-viewer, the knowing transgression of representational layers effected by the written descriptions on the "Come to Daddy" promo artwork. However, in so doing, he suggests that there may in fact be nothing more than the masks. By his status as the object of pixellation or vocal processing, James' own subjectivity is aligned with the non-subjective elements of his art. He "speaks" by ordering these masks, but we deduce this only by their presence, not by any underlying action on his part: the computer processing that mediates every element of the Richard D. James Album text creates the semblance of subjectivity, but there is no computer "self". By making the listener-viewer aware of this illusion, the Aphex Twin project invites speculation around where the machinery might end and the "real world" begin: James' handwriting, for example, is produced as the result of a pencil and muscular activity, which might be seen as machines in their own right.

\section{Conclusion [sic]}

Once again, the objection will be raised that music is not a language, that the components of sound are not pertinent features of language, that there is no correspondence between the two. [...] We keep asking that the issue be left open, that any presupposed distinction be rejected (Deleuze and Guattari 2004: 106).

I do not suggest that IDM is a language or a literature. Nonetheless, I wish to show that such a reading is more useful than attempts to see it purely as heir or constituent of existing musical traditions. Likewise, the incorporation of dialectical gestures into the argument should be seen as parallel to, rather than a component of, the Deleuzian methodology, although the two can be seen to mutually reinforce at certain points.

"Majority", write Deleuze and Guattari, "implies a constant, of expression or content, serving as a standard measure by which to evaluate it" (Deleuze and Guattari 2004: 116). IDM's treatment of this "standard measure" allows it to be seen as a "becoming-minor" of the majorities of intelligence, of dance, even of music: IDM does not resist these concepts in favour of others, but places in "continuous variation" (Deleuze and Guattari 2004: 116) elements of music and the music industry such that genre, subjectivity, canon, notation, and so forth are seen to be potentials, not constants.

There are not, therefore, two kinds of languages but two possible treatments of the same language. Either the variables are treated in such a way as to extract from them constants and constant relations or in such a way as to place them in continuous variation (Deleuze and Guattari 2004: 116).

In the present discussion, these "variables" extend to function, record format, the subject, and the relationship between remixer and remixed. As I have argued, IDM's treatment of these variables is not to see them as tendencies towards a particular norm but to maintain their variation. The examples of IDM that constitute deviations from musical value systems reveal such systems to be circular and arbitrary. EP7, in being longer than LP5, is not simply identified as transgressive: rather, artistic form is placed 
in continuous variation such that no single distinction between EP and LP can be "correct". Furthermore, instead of treating these potentials ironically - for instance, by assigning $E P 7$ a long-playing catalogue number - the literature surrounding the musical texts challenges the listener-viewer to evaluate such normative systems on the basis of utility, as opposed to any "underlying essence" from which they purport to derive authority. Artistry is located in the recording artist one moment, in the remixer the next, in the technical means to the product a third time. None of these is dominant; no locus for the author is treated as a constant by which the other loci are evaluated as deviant.

IDM is not isolated: its texts are frequently appropriative. What affords it its minority is its refusal to read the significance of the appropriated values (identity, subjectivity, function) normatively: the texts that apparently conform to them do so for no reason beyond their own ends. Richard D. James Album introduces its author's name to inform the play around subjectivity, to place authorship in "continuous variation", not to appeal to authority. Warp 10 appears to locate Warp in a narrative, but its contents are deterritorialising, not affirmative.

Deleuze and Guattari (2004: 106-107) prefigure McLuhan's suggestion that all sound has at least the potential to operate in "acoustic" space. This acoustic potential is indexed by IDM's inconsistent, non-hierarchical location of the musical text: the treatments of language and form by IDM's "authors" are such that any constants temporarily abstracted have no overall significance because there is no authority to sanction them (for which reason it makes no sense to speak of IDM "as a whole"). By "authors", I refer not just to the people whose names appear on the record sleeves; record labels, journalists, and theorists can all be seen to author the IDM "text". As Cox and Warner note in the introduction to 2004's Audio Culture anthology, the "heterogeneity [of the book's sources] reflects the fact that the new audio culture is a discourse, a loose collection of terms, concepts, and statements gathered from across the cultural field" (Cox and Warner 2007: xv).

In Deleuzian terms, IDM's authors are "foreigners in their own tongue" (Deleuze and Guattari 2004: 116) - dance artists who make records that do not have to be danced to, artists whose artistry is in a state of flux with relation to those who remix their songs, labels operating against the tendency to keep catalogue, draw constants, or reference canon. The effect of this work is to place the "major language" - the language of intelligence, of dance, of music, of form, of subjectivity, of canon - into continuous variation so that, for example, the mechanical subject and the mechanical object can exist on equal terms, neither of them claiming any single essence of what IDM is or is not.

One must find the minor language [...] on the basis of which one can make one's own major language minor. That is the strength of authors termed "minor," who are in fact the greatest, the only greats; having to conquer one's own language [...] in order to place it in a state of continuous variation (Deleuze and Guattari 2004: 116).

It is in the nature of these propositions that reduction or conclusion is not entirely appropriate, so I wish to end instead with the suggestion that the present work might be usefully continued by further studies of IDM's interactions with posthumanism and the $\mathrm{S} /$ subject, and by the examination of a far greater number of individual texts than has here been possible. Finally, it is important that these points themselves do not 
appear to construct a "true" IDM identity, even negatively. As Deleuze and Guattari (2004: 113) point out,

it is difficult to see how the upholders of a minor language can operate if not by giving it (if only by writing in it) a constancy and homogeneity making it a locally major language [...] But the opposite argument seems more compelling: the more a language has or acquires the characteristics of a major language, the more it is affected by continuous variations that transpose it into a "minor" language.

Above all, there can be no superstructure to which to appeal for IDM's conclusive inscription. The discussion neither begins nor ends here.

\section{References}

Adorno, Theodor W. 1988 [1955]. "The Aging of the New Music." Trans. Robert HullotKentor. Telos 77: 95-116.

Balliger, Robin. 1999. "Politics." In Bruce Horner and Thomas Swiss (eds), Key Terms in Popular Music and Culture, pp. 57-70. Oxford: Blackwell.

Colebrook, Claire. 2002. Gilles Deleuze. London: Routledge.

Cowley, Julian. 2002. "The Limits of Language." In Rob Young (ed), Undercurrents: The Hidden Wiring of Modern Music, pp. 193-208. London: Continuum.

Davis, Erik. 2008. "Roots and Wires' Remix: Polyrhythmic Tricks and the Black Electronic." In Paul D. Miller AKA DJ Spooky That Subliminal Kid (ed), Sound Unbound, pp. 53-72. London: The MIT Press.

Deleuze, Gilles and Félix Guattari. 2004 [1980]. A Thousand Plateaus. Trans. Brian Massumi. London: Continuum.

James, Richard D. and Marc Weidenbaum. 2001. "Eponymous Rex Interview." http://www.aphextwin.nu/learn/98136333384401.shtml (accessed 25 February 2009).

McLuhan, Marshall. 2007. "Visual and Acoustic Space." In Christoph Cox and Daniel Warner (eds), Audio Culture: Readings in Modern Music, pp. 67-72. London: Continuum.

Official UK Charts Company, The. 2009. "Chart Rules." http://theofficialcharts.com/chart_rules.php (accessed 7March 2009).

Park, Adam. 2008a. "Autechre - Quaristice." Clash Music, 6 February 2008. http://www.clashmusic.com/feature/autechre-quaristice (accessed 23 November 2008).

----. 2008b. "Autechre." Clash 25, April: 72-73.

Parry, Alan Michael, posting on alt.rave on 8 August 1993:

http://groups.google.com/group/alt.rave/browse_thread/thread/b8592fef4a3al4b4/af70a9963a500 2ddd \# af 70 a963a5002ddd (accessed 8 November 2008).

Porschardt, Ulf. 1998. DJ Culture. London: Quartet.

Reynolds, Simon. 1999. Generation Ecstasy. New York: Routledge.

Schopenhauer, Arthur. 1988 [1818]. Extract from Die Welt als Wille und Vorstellung. Trans. In Peter le Huray and James Day (eds), Music and Aesthetics in the Eighteenth and EarlyNineteenth Centuries, pp. 217-224. Cambridge: Cambridge University Press.

Shapiro, Peter. "Automating the Beat." In Rob Young (ed) Undercurrents: The Hidden Wiring of Modern Music, pp. 131-140. London: Continuum.

Shepherd, John. 1999. “Text." In Bruce Horner and Thomas Swiss (eds), Key Terms in Popular Music and Culture, pp. 156-177. Oxford: Blackwell. 
Stockhausen, Karlheinz, et al. 2007. "Stockhausen vs. the 'Technocrats." In Christoph Cox and Daniel Warner (eds), Audio Culture: Readings in Modern Music, pp. 381-385. London: Continuum.

Straw, Will. 1999. "Authorship." In Bruce Horner and Thomas Swiss (eds), Key Terms in Popular Music and Culture, pp. 199-208. Oxford: Blackwell.

Stubbs, David. 2003. "Autechre: The Futurologists." The Wire 230, April: 28-33.

Young, Rob. 2006. Warp: Labels Unlimited. London: Black Dog Publishing.

\section{Discography}

Where the discussion has referred to a release in general rather than any of its specific formats, I have defaulted below to the details of its commercial British LP or 12-inch version. Details of other formats appear when the formats themselves are discussed in the main text (as with "Come to Daddy"). Superscripts 1 to 8 mark the original Artificial Intelligence series in order of release.

Aphex Twin. 1994. Selected Ambient Works Volume II. Warp (2xLP): WARP LP21. http://www.discogs.com/Aphex-Twin-Selected-Ambient-Works-Volume-II/release/704310

----. 1996. "Come to Daddy". Warp (12-inch): WAP 94.

http://www.discogs.com/Aphex-Twin-Come-To-Daddy/release/3662

----. 1996. "Come to Daddy". Warp (2x12-inch promo): WAP 94RP, WAP 94P.

http://www.discogs.com/Aphex-Twin-Come-To-Daddy/release/28699

----. 1996. Richard D. James Album. Warp (LP): WARP LP43.

http://www.discogs.com/Aphex-Twin-Richard-D-James-Album/release/30849

Autechre. 1993. Incunabula. Warp (2xLP): WARP LP17. http://www.discogs.com/Autechre-Incunabula/release/29900

----. 1995. Garbage. Warp (12-inch): WARP 58. http://www.discogs.com/Autechre-Garbage/release/2498

----. 1997. Chiastic Slide. Warp (2xLP): WARP LP49 http://www.discogs.com/Autechre-Chiastic-Slide/release/19628

----. 1997. Cichlisuite. Warp (2x12-inch): WAP 96, WAP 96R. http://www.discogs.com/Autechre-Cichlisuite/release/28703 http://www.discogs.com/Autechre-Cichlisuite/release/28704

----. 1998. LP5. Warp (2xLP): WARP LP66.

http://www.discogs.com/Autechre-LP5/release/30811

----. 1999. EP7. Warp (2x12-inch): WAP EP7.1, WAP EP7.2.

http://www.discogs.com/Autechre-EP71/release/24545

http://www.discogs.com/Autechre-EP72/release/24544

----. 2008. Quaristice. Warp (2xLP): WARP LP333.

http://www.discogs.com/Autechre-Quaristice/release/1257383

----. 2008. Quaristice. Warp (FLAC download): WARP CDD333F.

http://www.discogs.com/Autechre-Quaristice/release/1864034

B12. 1993. Electro-Soma. Warp (2xLP): WARP LP9. http://www.discogs.com/B12-Electro-Soma/release/29889

Black Dog Productions. 1993. Bytes. Warp (2xLP): WARP LP8. http://www.discogs.com/Black-Dog-Productions-Bytes/release/4663

FUSE. 1993. Dimension Intrusion. Warp (2xLP): WARP LP12. http://www.discogs.com/FUSE-Dimension-Intrusion/release/38940

Future Sound of London, The. 1994. Lifeforms. Virgin (2xLP): V 2722. http://www.discogs.com/Future-Sound-Of-London-Lifeforms/release/83318 
Harmonic 313. 2009. When Machines Exceed Human Intelligence. Warp (2xLP): WARP LP175.http://www.discogs.com/Harmonic-313-When-Machines-Exceed-Human-Intelligence/rele ase $/ 1773959$

Kraftwerk. 1974. Autobahn. Vertigo (LP): 6360620. http://www.discogs.com/Kraftwerk-Autobahn/release/63961

LFO. 1991. Frequencies. Warp (LP): WARP LP3. http://www.discogs.com/LFO-Frequencies/release/29366

Pink Floyd. 1973. The Dark Side of the Moon. Harvest (LP): SHVL 804. http://www.discogs.com/Pink-Floyd-The-Dark-Side-Of-The-Moon/release/371269

Polygon Window. 1993. Surfing on Sine Waves. Warp (2xLP): WARP LP7. http://www.discogs.com/Polygon-Window-Surfing-On-Sine-Waves/release/ 18360

Speedy J. 1993. Pepper. Warp (2xLP): WARP LP14. http://www.discogs.com/Speedy-J-Ginger/release/29894

Various. 1991. Pioneers of the Hypnotic Groove. Warp (LP): WARP LP2. http://www.discogs.com/Various-Pioneers-Of-The-Hypnotic-Groove/release/29365

Various. 1992. Artificial Intelligence. Warp (LP): WARP LP6. http://www.discogs.com/Various-Artificial-Intelligence/release/29372

Various. 1994. Artificial Intelligence II. Warp (2xLP): WARP LP23. http://www.discogs.com/Various-Artificial-Intelligence-II/release/2966

Various. 1999. Warp 10+1 Influences. Warp (4xLP): WARP LP67. http://www.discogs.com/Various-Warp-101-Influences/release/30833

Various. 1999. Warp 10+2 Classics 89-92. Warp (4xLP): WARP LP68. http://www.discogs.com/Various-Warp-102-Classics-89-92/release/6551

Various. 1999. Warp 10+3 Remixes. Warp (4xLP): WARP LP69. http://www.discogs.com/Various-Warp-103-Remixes/release/30834

\section{Author Biography}

Ramzy Alwakeel was born in 1988 in Leicester in the East Midlands of England. He graduated from the University of Leeds in July 2009 with a First Class Honours degree in Music, and currently lives and works in Manchester.

\section{Notes}

1 “ 313 " bears more than a passing resemblance to [TB] " 303 ", the Roland bass synth that essentially revolutionised dance music; 313 is also Detroit's dialling code, which identifies artist with mechanical mediation in a further sense.

2 A further effect is performed by the designation of the 12-inch, not the 7 -inch or the LP, as the locus of these tracks' identities, despite the focus on albums over singles outlined above.

3 "Fold4,Wrap5" is a track on LP5 whose shifts in rhythmic focus and constant deceleration rather give it the effect of a sonic Escher painting.

$4 \quad$ LP5 itself is so called by convention: nowhere on the artwork is a title other than the word "Autechre" given. Warp, however, lists the release as LPS on their official website under "albums". The catalogue number WARP LP66 further indicates that LP5 is to be specifically read as an album.

5 Mute Records, for example, issue their LPs with the code STUMM XXX, and add prefixes to catalogue other formats.

6 "IDM" puts easily as much sonic focus upon timbre, parameters and envelopes as upon the "parts" that might be identified by a more classical analysis. 